\title{
Long-lasting neurocognitive disorders: a case report of previously undescribed adverse effects after ketamine sedation and analgesia in a pediatric patient
}

\author{
Kissila M. Machado-Ferraro, ${ }^{1,2,3 \#}$, Débora S. Soriano-de-Mello ${ }^{4 \#}$, Isadora P. de Moura ${ }^{4 \#,}$ \\ Cinthia C. S. M. da Silveira ${ }^{3 \wedge}$, Emmerson C. F. de Farias ${ }^{2} \wedge$, Mary L. F. Maia ${ }^{1,2}$, Susan C. D. de Sales ${ }^{2}$, \\ Ana Emilia V. Carvalho ${ }^{4}$, Ismaelino M. N. Magno ${ }^{4} \wedge$, Enéas A. Fontes-Júnior ${ }^{3 \wedge}$, Cristiane S. F. Maia ${ }^{1,3} \wedge$ \\ ${ }^{1}$ Post-Graduate Program in Pharmacology and Biochemistry, Federal University of Pará, Belém, Pará, Brazil; ${ }^{2}$ Hospital Fundação Santa Casa de \\ Misericórdia do Pará (FSCMP), Unidade Materno Infantil Dr. Almir Gabriel, Belém, Pará, Brazil; ${ }^{3}$ Laboratory of Pharmacology of Inflammation \\ and Behavior, Faculty of Pharmacy, Health Science Institute, Federal University of Pará, Belém, Pará, Brazil; ${ }^{4}$ University Center of Pará-CESUPA, \\ Belém, Pará, Brazil \\ \#These authors contributed equally to this work. \\ Correspondence to: Dr. Cristiane S. F. Maia. Programa de Pós-Graduação em Farmacologia e Bioquímica, Universidade Federal do Pará, Rua Augusto \\ Corrêa 01, Campus Universitário do Guamá, Belém, Pará, Brazil. Email: crismaia@ufpa.br.
}

\begin{abstract}
This case report describes the long-term behavioral and cognitive alterations in a critically ill pediatric patient submitted to a ketamine sedation and analgesia protocol for 7 consecutive days in a pediatric intensive care unit. The infant exhibited withdrawal syndrome in the early withdrawal period, as measured using the Withdrawal Assessment Tool-1 (WAT-1). In the days following ketamine withdrawal, behavioral, motor, and cognitive impairment was observed, even after hospital discharge. At 20 days after admission to hospital, the infant still displayed language deficits compatible with the at-risk category for the appropriate age group on the development assessment (Denver-II Developmental Screening Test). The infant's mother reported that these impairments were not present before ketamine sedation. We therefore suggest that prolonged ketamine use may have contributed to the long-lasting behavioral and cognitive impairments observed in the critically ill infant. These adverse effects may be attributable to ketamine's pharmacological mechanism of action, by which the N-methyl-D-aspartate receptor-the central nervous system excitatory receptor responsible for memory and learning domains-is blockaded, disrupting long-term potentiation events. Our case highlights the need for clinical evaluation of ketamine agents and their associated risks in intensive care units to better clarify appropriate sedative and analgesic agents during neurodevelopmental periods of life.
\end{abstract}

Keywords: Ketamine; sedation; neurological disorders; behavioral impairment; case report

Submitted May 04, 2021. Accepted for publication Aug 13, 2021.

doi: $10.21037 /$ atm-21-2292

View this article at: https://dx.doi.org/10.21037/atm-21-2292

^ ORCID: Cinthia C. S. M. da Silveira, 0000-0003-0438-0525; Emmerson C. F. de Farias, 0000-0001-8627-5694; Ismaelino M. N. Magno, 00000003-3323-1465; Enéas A. Fontes-Júnior, 0000-0002-6186-9581; Cristiane S. F. Maia, 0000-0003-4493-7375. 


\section{Introduction}

Early childhood is a critical period in the development of cognitive and behavioral skills (1). During this developmental stage, the human brain undergoes fundamental morphological, volume, compositional, and functional changes which establish neural connection networks in the cognitive, motor, and sensory domains (2). Environmental factors, such as exposure to psychotropic drugs, may also influence brain maturation and impair some central nervous system functions (2).

In pediatric intensive care units (PICUs), the use of drugs that modify brain physiology is necessary to support critical procedures. Ketamine is the preferred drug for sedation and analgesia in children due to its bronchodilatory effects and cardiovascular stability resulting from the upregulation of catecholamines and the stimulation of the $\beta 2$-adrenergic receptors. This mechanism of action is beneficial for patients with respiratory comorbidities such as asthma, bronchospasm, or other disorders which impair cardiorespiratory homeostasis and require sedation. However, at high doses, ketamine can induce hallucinations and death of immature neurons (3).

Few studies to date have reported the consequences of ketamine sedation in PICUs. However, recreational use of ketamine at subsedative doses in adults has increasingly been reported to induce symptoms of delirium, hallucinations, and time and space distortion. Further, behavioral and cognitive impairments may persist even after long-term withdrawal (4). Therefore, it is plausible that sedative doses of ketamine during critical phases of brain development and maturation may induce long-lasting neurological disorders.

This paper reports the case of a critically ill infant who displayed behavioral and cognitive symptoms following a 7-day sedation and analgesia protocol with ketamine. The infant exhibited behavioral, motor, and cognitive impairments during the early withdrawal period, along with language deficits that persisted 20 days after hospital discharge. This unique report provides evidence of longlasting cognitive impairments resulting from ketamine sedation in an infant. Such impairments, which may jeopardize regular neurodevelopment and necessitate multiple follow-ups to detect potential consequences in adulthood, have often been neglected by healthcare teams and require further study.

This case study has been reported in line with the CARE reporting checklist (available at https://atm.amegroups. com/article/view//10.21037/atm-21-2292/rc).

\section{Case presentation}

All procedures performed in this study followed the Declaration of Helsinki (as revised in 2013). The study was approved by the Ethics Committee on Human Research from the Santa Casa de Misericórdia Foundation Hospital of Pará (FSCMP), Belém, Brazil (license no. 3.749.278). Written informed consent was obtained from the patient's legal representative.

An 18-month-old male infant with a body weight of $11.11 \mathrm{~kg}$ was admitted to the FSCMP on July 21,2020 . The infant's family reported a normal behavioral and cognitive profile and no previous hospitalizations. The infant had exhibited flu symptoms for 3 days before hospitalization, and a cough and respiratory discomfort during the preceding 24 hours. As several of the infant's relatives had COVID-19, he was empirically diagnosed with COVID-19 and kept under respiratory isolation in the PICU.

At admission, the infant presented with tachydyspnea and was using accessory muscles to breathe, even during oxygen therapy with mask ventilation. Owing to the presence of clinical symptoms of hemodynamic instability, orotracheal intubation and the hospital's sedation and analgesia protocol were performed. Both ketamine and midazolam were administered as a bolus dose during the initial attack dose (in the rapid sequence of intubation), after which a maintenance dose was given through continuous infusion (Table 1); this was the infant's first contact with psychoactive substances. On hospitalization day (D) 1, the infant exhibited a deterioration of clinical symptoms (radiological worsening and hypercapnia), so was placed in the prone position. Subsequently, the doses of the sedative drugs were doubled and the neuromuscular blocker rocuronium was administered to maintain a COMFORT behavior scale score of between 6 and 10 (with 5 indicating deep sedation) (5).

After 3 days, the infant's clinical and radiological conditions had improved. Rocuronium administration was withdrawn and ventilator weaning was initiated. On D5, dexmedetomidine was administered to reduce ketamine use. On D7, the infant developed ventilationassociated pneumonia; extubation was postponed and the administration of ketamine, midazolam, fentanyl, and dexmedetomidine was maintained. After 8 days of sedation, the ketamine administration was withdrawn, and psychomotor agitation, uncoordinated limb and tongue movements, and abdominal distension were observed, which translated to a score of 4 on the Withdrawal Assessment Tool-1 (WAT-1) and indicated a high probability of 
Table 1 Sedoanalgesic drugs, COMFORT B scale, and Withdrawal Assessment Tool 1 (WAT-1) versus hospitalization days (D) of the infant

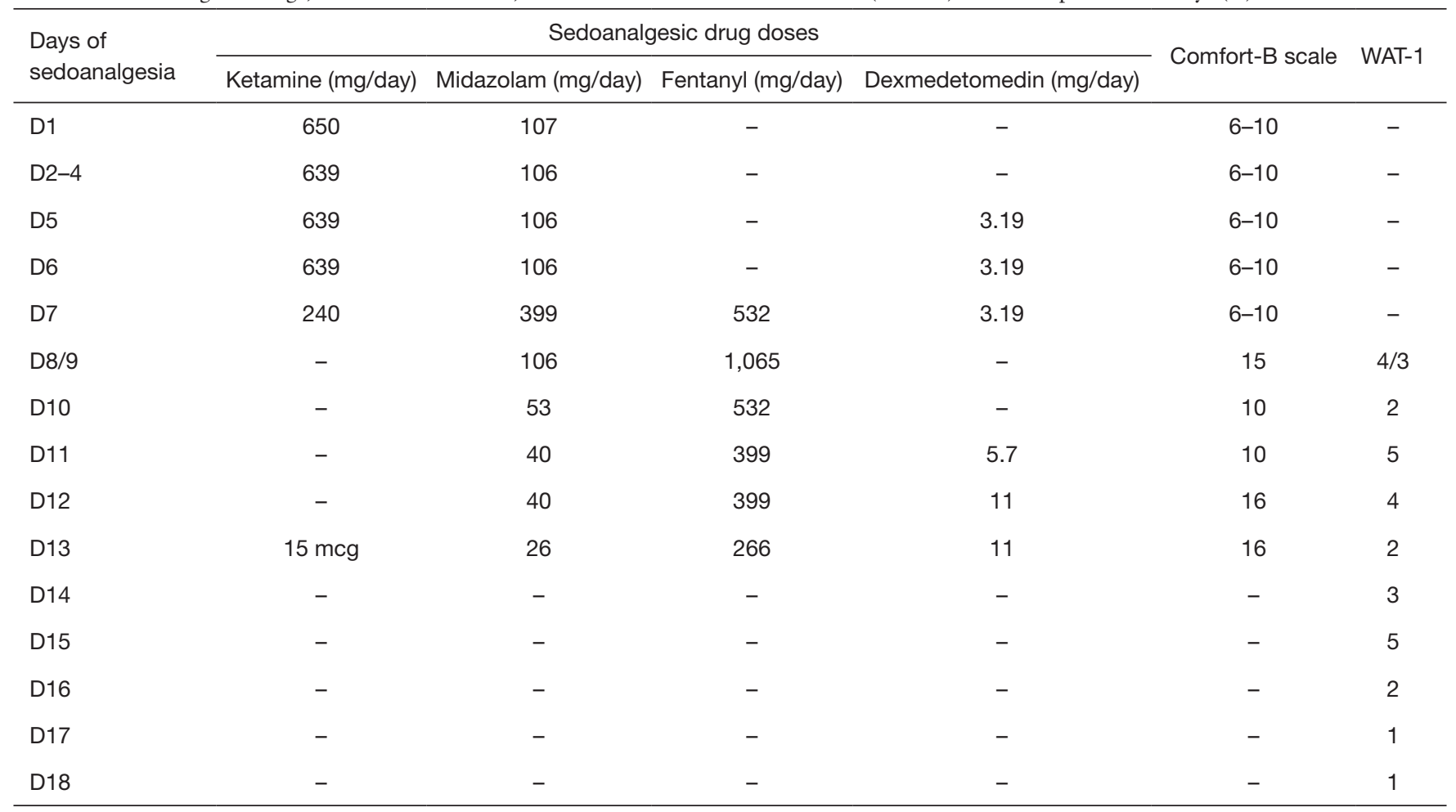

withdrawal syndrome (6). Even with the administration of methadone, chlorpromazine, clonidine, and dexmedetomidine, the infant had a WAT-1 score of at least 3 during the 5-day period (D8/9-D12) following ketamine withdrawal, with the exception of D10. Interestingly, on D11, the infant displayed the first abnormal agitation, characterized by recurrent motor stereotypy with body and head sway, which was refractory to sedative drugs and accompanied by a WAT-1 score of 5 and 4 on D11 and D12, respectively. Ketamine was therefore administered for 1 day to minimize this behavioral alteration. After the reintroduction of ketamine, the WAT-1 score fell to 2 on D13, before rising to 3 on D14.

After 14 days of hospitalization, mechanical ventilation was discontinued until extubation by following an abstinence protocol, with a WAT-1 score of between 3 and 5 (D14-15). Fever, sleep-wake inversion, and sialorrhea were observed. It is noteworthy that behavioral alterations, such as sleep-wake inversion, were still observed on D17, when the WAT-1 score had decreased to 1 . During physical examination, the infant exhibited tachycardia, vomiting, movement disorder (for which containment measures were necessary), and neuromotor dysfunctions affecting neck support and sitting posture. At 5 days after extubation (D19), the infant was not able to walk or verbalize.

At 5 days after the last ketamine administration (D18), the infant was discharged from the PICU and remained in the ward until hospital discharge on D22. The infant's sialorrhea and motor and attention impairments showed improvement, but sleep-wake inversion and neck support impairment could still be observed.

At the follow-up 20 days after hospital discharge, the infant's caregiver reported that he was displaying aggressiveness, irritability, sleep-wake inversion, crying, muscle weakness, and trembling limbs during sleep (similar to muscle spasms). Also, the infant had only started to walk and run again at 10 and 12 days after hospital discharge, respectively. The infant's development assessment suggested normal personal-social behavior, fine motor-adaptive skills, and gross motor skills. In terms of language, the infant was ranked as at risk for developmental delay based on the Denver II Developmental Screening Test (DDST-II). The infant presented with difficulties with respect to expressive and receptive vocabulary, which includes words such as dada/daddy, mama/mommy, and "wata"/water. More than $50 \%$ of the infant's expressive language was gestural (i.e., 


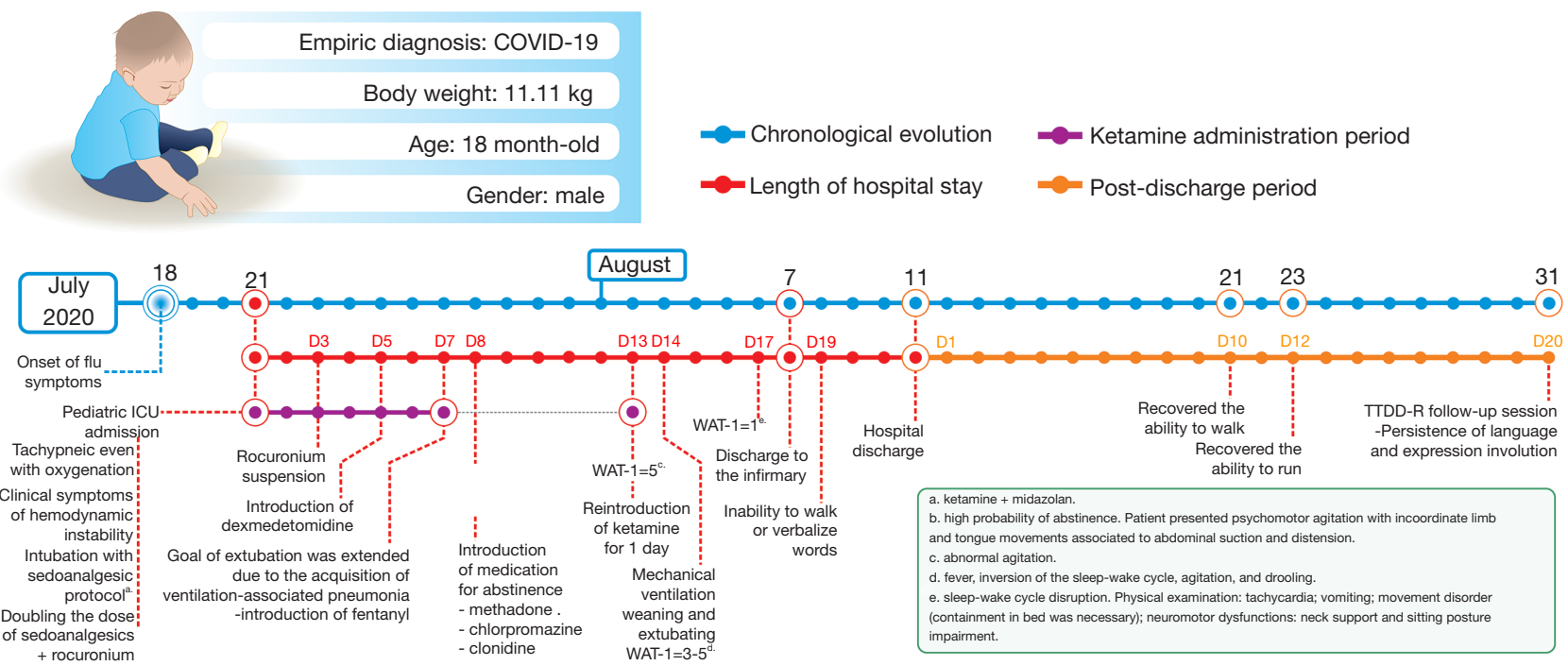

Figure 1 Schematic historical and current information from the episode of care.

pointing at the desired object) and unintelligible (i.e., unable to combine words to form sentences). MRI of the infant's skull uncovered no anatomical abnormalities or areas of restricted diffusion. The timeline of the infant's management and outcome is presented in Figure 1.

\section{Discussion}

Ketamine is a dissociative sedative with analgesic action that produces a psychological mood-control effect. Despite the scarcity of studies on the use of ketamine in the sedation of critically ill patients, the drug has been recommended for use in ICU procedures based on its protective effects involving the gastrointestinal and cardiorespiratory systems; although, it is worth noting that a pediatric multicenter study failed to detect a hemodynamic protective effect $(7,8)$. The long-term behavioral and cognitive effects of ketamine use in critically ill pediatric patients are unknown.

This paper has reported a case of unexpected behavioral and cognitive consequences observed in a critically ill infant submitted to sedation and analgesia in a PICU. The infant displayed a high probability of withdrawal syndrome (WAT1 score between 3 to 5 ) in the 24 to 48 hours following ketamine suspension (D14-15). Fever, sleep-wake inversion, agitation, and sialorrhea were observed, even with the maintenance of midazolam and fentanyl infusion. These undesired ketamine-induced behavioral side effects after sedation have been reported previously (9).

On D17, the infant was still exhibiting behavioral and cognitive disorder, including aggressiveness and language difficulties. The infant was therefore followed up at 20 days after hospital discharge to allow for the evaluation of behavioral and cognitive improvements in response to home environment stimuli. Interestingly, behavioral alterations affecting motor skills were observed for up to 2 weeks. The infant's mother reported that her son only started to walk and run again after 10 to 12 days at home. Cognitive impairments affecting language and expression could still be observed after 20 days following hospital discharge and approximately 1 month after ketamine administration. The DDST-II indicated that the infant was at risk for cognitive impairment with respect to his age group. This result raises the question of whether cognitive intervention will be necessary to improve the infant's learning curve and reduce negative effects in adolescence and adulthood.

The purpose of this case report was not to describe ketamine-induced side effects during PICU hospitalization, but rather to pose questions regarding the long-term consequences of ketamine use in the sedation and analgesia of infants. In particular, the present case prompts questions as to whether the therapeutic use of ketamine for sedation is beneficial considering its long-lasting behavioral consequences in the immature brain, and whether the difficulty in controlling ketamine and the pharmacokinetic profile of its active metabolites induces behavioral alterations in pediatric patients (10).

To date, the limited literature on sedation and analgesia in critically ill children has focused on ketamine's beneficial 
pharmacological effects (11), rather than its effects post use. Despite the multiple comorbidities that require treatment in the PICU, the role of ketamine neurotoxicity in poor neurodevelopmental outcomes relating to behavioral impairment has been reported in both animal and human studies (12). However, more robust data are needed to confirm the clinical relevance of these studies, as hypoxia, hemodynamic and respiratory instabilities, stress, and inflammation observed in hospitalized patients may also influence behavioral outcomes. Animal studies have revealed that sedative agents can trigger dendritic morphology alterations and neuronal apoptosis due to their blockade of N-methyl-D-aspartate (NMDA) receptors in a dose-dependent manner, in which the dose, period of exposure, and window of brain vulnerability are relevant factors (13). Synaptic plasticity refers to the ability to alter the connections between neurons to favor learning and memory. This neuronal process is dependent on long-term potentiation events, which in turn depend on NMDA signaling triggering cognitive functions (14). Pharmacological agents that interfere in the NMDA circuitry may thus affect cognitive domains (12).

Our research group recently demonstrated that ketamine administration at subanesthetic doses in adolescent rats for 3 consecutive days induced negative behavioral consequences and cognitive deficits associated with hippocampal oxidative damage in the early hours of ketamine withdrawal (15). Moreover, experimental studies have reported negative long-term behavioral consequences (i.e., learning and spontaneous activity) after a single dose of anesthetic agents (including ketamine) (16). A cohort study found that children who experienced multiple exposures to anesthesia before the age of 4 years exhibited learning disabilities later in life (17). Ketamine-induced neurotoxicity has been associated with several mechanisms involving the non-competitive antagonism of NMDA glutamatergic receptors, such as the reduction of protein kinase $\mathrm{C}$ (PKC)/extracellular signal-related kinase (ERK) pathway phosphorylation, LTP inhibition, and reduced expression of anti-apoptotic factors, such as the B-cell lymphoma 2 (Bcl-2) protein (18). Also, in the late stage following the blockade of NMDA receptors, the NMDA receptor is further upregulated and triggers glutamate excitotoxicity. The intense calcium influx activates multiple degenerative mechanisms such as protease, neuroinflammation, mitochondrial dysfunction, and oxidative stress (19), which may result in long-lasting cognitive deficits and learning disabilities in developing brains (Figure 2).

The neurocognitive impairments reported in the present case may be multifactorial. However, the clinical evidence related to the use of other sedative drugs, such as alpha-2 adrenergic (e.g., dexmedetomidine), gamma-aminobutyric acid (e.g., midazolam), and opioid receptor (e.g., fentanyl) agonists, and their pharmacological mechanisms of action, cannot explain the long-term cognitive disabilities reported (20). The fact that our infant was submitted to sedation and analgesia with ketamine for 7 consecutive days, including an additional infusion on D13, suggests that prolonged exposure to ketamine contributed to his behavioral alterations. Further, the structured questionnaire completed by the infant's mother prior to hospitalization confirmed that his cognitive and language skills were adequate and that the impairments observed after hospital discharge were not the pre-ketamine behaviors of the child.

In summary, prolonged sedation and analgesia with ketamine and other drugs induced motor, behavioral, and cognitive alterations in a critically ill infant during the early period of ketamine withdrawal. The infant's motor skills damage recovered after 10 days of ketamine abstinence, whereas behavioral and cognitive effects, such as sleepwake inversion and language deficits, could still be observed 20 days after hospital discharge. This case highlights the neurodevelopmental vulnerability of individuals submitted to the administration of sedative agents. Further controlled clinical studies of long-term neurobehavioral alterations that may affect individuals in adulthood are necessary for the establishment of evidence-based sedation and analgesia protocols in infants. 


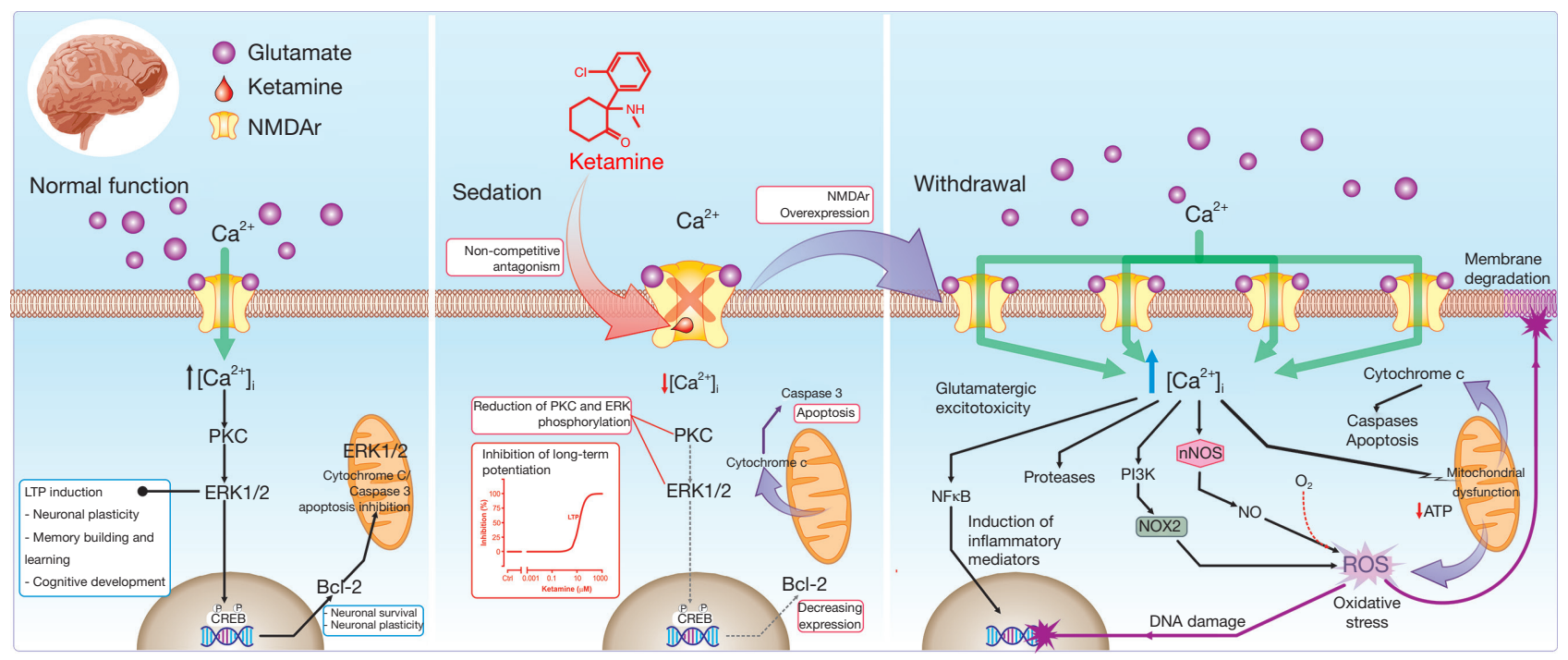

Figure 2 Ketamine-induced developmental neurotoxicity mechanism. Prolonged pediatric exposure to ketamine may produce longterm neurocognitive deficits. Several mechanisms have been proposed to elucidate this effect, all of which are associated with the drug's non-competitive antagonism of N-Methyl-D-Aspartate (NMDA) glutamatergic receptors. Through blockade of the NMDA receptor (NMDAR), which is the main mechanism of sedative activity, a reduction in protein kinase C (PKC)/extracellular signal-related kinase (ERK) phosphorylation, long-term potentiation (LTP) inhibition, and reduced expression of anti-apoptotic factors, such as the B-cell lymphoma 2 (Bcl-2) protein, can be observed. Another consequence is upregulation of the NMDAR, which, after prolonged ketamine use, triggers the occurrence of glutamatergic excitotoxicity. The intense influx of calcium $\left(\mathrm{Ca}^{2+}\right)$ activates multiple degenerative mechanisms, including protease, neuroinflammation, mitochondrial dysfunction and oxidative stress. In developing brains, these conditions could be the basis for cognitive deficits and learning disabilities, and have long-term repercussions.

\section{Acknowledgments}

We would like to thank Santa Casa de Misericórdia do Pará and Centro Universitário do Pará-CESUPA.

Funding: This work was supported by a Research Productivity Grant awarded to Dr. Cristiane do Socorro Ferraz Maia by the Conselho Nacional de Desenvolvimento Científico e Tecnológico - CNPq/ Brazil CNPq (311335/2019-5) and by the Research Pro-Rectory of the Federal University of Pará (PROPESP, UFPA, Brazil), which provided the article publication fee.

\section{Footnote}

Reporting Checklist: The authors have completed the CARE reporting checklist. Available at https://atm.amegroups. com/article/view//10.21037/atm-21-2292/rc

Peer Review File: Available at https://atm.amegroups.com/ article/view//10.21037/atm-21-2292/prf

Conflicts of Interest: All authors have completed the
ICMJE uniform disclosure form (available at https://atm. amegroups.com/article/view//10.21037/atm-21-2292/coif). The authors have no conflicts of interest to declare.

Ethical Statement: The authors are accountable for all aspects of the work in ensuring that questions related to the accuracy or integrity of any part of the work are appropriately investigated and resolved. All procedures performed in this study followed the Declaration of Helsinki (as revised in 2013). The study was approved by the Ethics Committee on Human Research from the Santa Casa de Misericórdia Foundation Hospital of Pará (FSCMP), Belém, Brazil (license No. 3.749.278). Written informed consent was obtained from the patient's legal representative for publication of this case report and accompanying images. A copy of the written consent is available for review by the editorial office of this journal.

Open Access Statement: This is an Open Access article distributed in accordance with the Creative Commons Attribution-NonCommercial-NoDerivs 4.0 International 
License (CC BY-NC-ND 4.0), which permits the noncommercial replication and distribution of the article with the strict proviso that no changes or edits are made and the original work is properly cited (including links to both the formal publication through the relevant DOI and the license). See: https://creativecommons.org/licenses/by-nc-nd/4.0/.

\section{References}

1. Gilmore JH, Knickmeyer RC, Gao W. Imaging structural and functional brain development in early childhood. Nat Rev Neurosci 2018;19:123-37.

2. Crews F, He J, Hodge C. Adolescent cortical development: a critical period of vulnerability for addiction. Pharmacol Biochem Behav 2007;86:189-99.

3. Bhutta AT. Ketamine: a controversial drug for neonates. Semin Perinatol 2007;31:303-8.

4. Morgan CJ, Muetzelfeldt L, Curran HV. Consequences of chronic ketamine self-administration upon neurocognitive function and psychological wellbeing: a 1-year longitudinal study. Addiction 2010;105:121-33.

5. Saelim K, Chavananon S, Ruangnapa K, et al. Effectiveness of Protocolized Sedation Utilizing the COMFORT-B Scale in Mechanically Ventilated Children in a Pediatric Intensive Care Unit. J Pediatr Intensive Care 2019;8:156-63.

6. Franck LS, Scoppettuolo LA, Wypij D, et al. Validity and generalizability of the Withdrawal Assessment Tool-1 (WAT-1) for monitoring iatrogenic withdrawal syndrome in pediatric patients. Pain 2012;153:142-8.

7. Ostermann ME, Keenan SP, Seiferling RA, et al. Sedation in the intensive care unit: a systematic review. JAMA 2000;283:1451-9.

8. Tarquinio KM, Howell JD, Montgomery V, et al. Current medication practice and tracheal intubation safety outcomes from a prospective multicenter observational cohort study. Pediatr Crit Care Med 2015;16:210-8.

9. Rita L, Seleny FL. Ketamine hydrochloride for pediatric premedication. II. Prevention of postanesthetic excitement. Anesth Analg 1974;53:380-2.

10. Flint RB, Brouwer CNM, Kränzlin ASC, et al. Pharmacokinetics of S-ketamine during prolonged sedation at the pediatric intensive care unit. Paediatr Anaesth 2017;27:1098-107.

11. Park S, Choi AY, Park E, et al. Effects of continuous ketamine infusion on hemodynamics and mortality in critically ill children. PLoS One 2019;14:e224035.

12. Davidson AJ. Anesthesia and neurotoxicity to the developing brain: the clinical relevance. Paediatr Anaesth 2011;21:716-21.

13. Slikker W Jr, Zou X, Hotchkiss CE, et al. Ketamineinduced neuronal cell death in the perinatal rhesus monkey. Toxicol Sci 2007;98:145-58.

14. Cain DP. LTP, NMDA, genes and learning. Curr Opin Neurobiol 1997;7:235-42.

15. de Carvalho Cartágenes S, Fernandes LMP, Carvalheiro TCVS, et al. "Special K" Drug on Adolescent Rats: Oxidative Damage and Neurobehavioral Impairments. Oxid Med Cell Longev 2019;2019:5452727.

16. Fredriksson A, Pontén E, Gordh T, et al. Neonatal exposure to a combination of $\mathrm{N}$-methyl-D-aspartate and gamma-aminobutyric acid type A receptor anesthetic agents potentiates apoptotic neurodegeneration and persistent behavioral deficits. Anesthesiology 2007;107:427-36.

17. Wilder RT, Flick RP, Sprung J, et al. Early exposure to anesthesia and learning disabilities in a population-based birth cohort. Anesthesiology 2009;110:796-804.

18. Jiang S, Li X, Jin W, et al. Ketamine-induced neurotoxicity blocked by N-Methyl-d-aspartate is mediated through activation of PKC/ERK pathway in developing hippocampal neurons. Neurosci Lett 2018;673:122-31.

19. Zhang Y, Sha R, Wang K, et al. Protective effects of tetrahydropalmatine against ketamine-induced learning and memory injury via antioxidative, anti-inflammatory and anti-apoptotic mechanisms in mice. Mol Med Rep 2018;17:6873-80.

20. Cruickshank M, Henderson L, MacLennan G, et al. Alpha-2 agonists for sedation of mechanically ventilated adults in intensive care units: a systematic review. Health Technol Assess 2016;20:v-xx, 1-117.

(English Language Editors: C. Gourlay and J. Reynolds)

Cite this article as: Machado-Ferraro KM, Soriano-de-Mello DS, de Moura IP, da Silveira CCSM, de Farias ECF, Maia MLF, de Sales SCD, Carvalho AEV, Magno IMN, Fontes-Júnior EA, Maia CSF. Long-lasting neurocognitive disorders: a case report of previously undescribed adverse effects after ketamine sedation and analgesia in a pediatric patient. Ann Transl Med 2022;10(2):113. doi: 10.21037/atm-21-2292 